\title{
Regulation of anti-inflammatory cytokines IL-10 and TGF- $\beta$ in mouse dendritic cells through treatment with Clonorchis sinensis crude antigen
}

\author{
Yan Jin, Hae Joo Wi, Min-Ho Choi, Sung-Tae Hong and Young Mee Bae
}

Dendritic cells (DCs), which are regarded as the most potent antigen-presenting cells, are involved in innate and adaptive immunity. Upon uptake of pathogens, DCs express cell surface markers and secrete cytokines. In this study, we analyzed production of cytokines and found that interleukin (IL)-10 and transforming growth factor (TGF)- $\beta$ production significantly increased in bone marrow-derived DCs and a mouse DC line, DC2.4, after treatment with crude antigen (CA) from liver fluke, Clonorchis sinensis. However, expression patterns of several activation molecules did not change. In addition, following treatment of DC2.4 cells with antigen from the lung fluke, Paragonimus westermani, production of IL-10 and TGF- $\beta$ significantly increased compared with groups treated with other parasite antigens, Spirometra erinacei plerocercoid CA and Echinococcus granulosus hydatid cystic fluid. We also found that treatment of DC2.4 cells with $C$. sinensis CA resulted in rapid and significant phosphorylation of extracellular signal-regulated kinase 1/2, a mitogen-activated protein kinase. Following treatment of DC2.4 cells with $C$. sinensis CA, treatment with an inhibitor specific to an extracellular signal-regulated kinase inhibited production of IL-10 and TGF- $\beta$. Our results suggest that CA from $C$. sinensis has a role in the anti-inflammatory function of DC cells by inducing IL-10 and TGF- $\beta$ through activation of extracellular signal-regulated kinase $1 / 2$. Experimental \& Molecular Medicine (2014) 46, e74; doi:10.1038/emm.2013.144; published online 31 January 2014

Keywords: Clonorchis sinensis; cytokine; dendritic cells; ERK1/2; immune regulation

\section{INTRODUCTION}

Dendritic cells (DCs) are the main antigen-presenting cells that are important in balancing immune responses between tolerance induction and immune activation, stimulating primary immune responses and driving inflammatory responses associated with immunopathology. ${ }^{1}$ For efficient antigen presentation to naive $\mathrm{T}$ cells, immature $\mathrm{DCs}$ are well positioned throughout the body to recognize and capture invading pathogens through pattern-recognizing receptors. ${ }^{1}$ The process of DC activation is accompanied by enhanced expression of co-stimulatory molecules such as CD40, CD80 and CD86, and major histocompatibility complex class II molecules. $^{2}$ Many pathogens, including parasitic worms (helminthes), induce highly polarized Th2 and T-regulatory cell immune responses. ${ }^{3}$ Although much is known about mechanisms that have evolved in bacteria and viruses to evade or use host defense mechanisms to avoid immune clearance, little is known about these mechanisms in helminthes. Nevertheless, studies indicate that these mechanisms differ considerably among these organisms by their DC activation status. ${ }^{4}$

Recently, subsets of regulatory DCs were identified and considered to be important in maintaining immune homeostasis. Regulatory DCs negatively regulate immune responses by inducing generation of regulatory $\mathrm{T}$ (Treg) cells or a preferential Th2 response. Regulatory DCs also display a Th2-biased cytokine profile by secreting increased levels of interleukin (IL)-10 and decreased levels of IL-12p70.5 However, the molecular mechanisms involved in Th2-biased cytokine production in regulatory DCs remains unclear.

Helminths have evolved means of evading or suppressing inflammatory responses. ${ }^{6,7}$ In particular, helminth products are antigenic, demonstrate immunomodulatory properties, and have been shown to suppress DC maturation and function when stimulated with Toll-like receptor (TLR) ligands. ${ }^{8}$ For these reasons, helminthes protect against the

Department of Parasitology and Tropical Medicine, Seoul National University College of Medicine and Institute of Endemic Diseases, Seoul National University Medical Research Center, Seoul 110-799, Korea

Correspondence: Professor YM Bae, Department of Parasitology and Tropical Medicine, Seoul National University College of Medicine and Institute of Endemic Diseases, Seoul National University Medical Research Center, 28 Yeongeon-dong, Jongro-gu, Seoul 110-799, Korea. 
development of autoimmunity and allergy. ${ }^{9}, 10$ For example, the filarial nematode-secreted product ES-62 has been shown to have a wide range of immunomodulatory properties. ${ }^{11,12}$ ES-62 can modulate immune responses via interactions with $B$ cells, macrophages and DCs. In particular, ES-62 was shown to significantly suppress lipopolysaccharide (LPS)-induced production of IL-12p40 and IL-12p70 in DCs ${ }^{11}$ and IL-12p40, IL-12p70, TNF- $\alpha$ and IL-6 production in macrophages. ${ }^{12}$ Similarly, Schistosoma mansoni egg antigen significantly suppressed TLR ligand-induced production of IL-12p40 and IL-12p70 in DCs in vitro and enhanced LPS-induced production of IL-10. ${ }^{13}$ Fasciola-derived antigens have also been shown to suppress antigen-specific Th1 immune responses in vitro and in vivo. ${ }^{14}$

Clonorchis sinensis Looss, 1907, is a Chinese liver fluke that dwells in the biliary tract of humans, dogs, cats, pigs and minks. C. sinensis infections are common in eastern parts of Asia such as China, Vietnam, Taiwan, East Russia and Korea. At least 35 million people have been infected with $C$. sinensis in endemic areas all over the world. ${ }^{15}$ On the basis of results showing increases in positive rates for $C$. sinensis eggs compared with other intestinal parasites in Korea, national surveys on the prevalence of intestinal parasitic infections have been carried out every $5-7$ years. ${ }^{16}$

C. sinensis is a parasitic helminth and a causative agent of clonorchiasis in humans in endemic areas. When mice are infected with C. sinensis, levels of Th2 cytokines (IL-4, IL-5 and IL-10) increase, whereas levels of Th1 cytokines (interferon- $\gamma$ and IL-12) decrease, ${ }^{17}$ suggesting that $C$. sinensis induces a dominant Th2/T-regulatory-type immune response. However, the effects of $C$. sinensis on DC function have yet to be determined. In this study, we prepared crude antigen (CA) from $C$. sinensis, and we are the first to investigate the effects of $C$. sinensis CA on DC maturation and cytokine production.

Until now, the influence of several helminth antigens from filarial nematode-secreted products, such as ES-62, S. mansoni egg antigen and Fasciola hepatica tegment antigen, on DCs had been determined. However, because the effect of $C$. sinensis on DCs has not been reported, we studied the effect of $C$. sinensis CA on DCs.

Here, we report that upon addition of $C$. sinensis CA, DCs preferentially secrete IL-10 and transforming growth factor (TGF)- $\beta$, but not other proinflammatory cytokines, through regulation of the ERK pathway. These results imply that secretion of regulatory cytokines IL-10 and TGF- $\beta$ may have an important role in the strict regulation of immune responses, at the APC level, in a feedback manner.

\section{MATERIALS AND METHODS}

\section{Preparation of $C$. sinensis CA}

Pseudorasbora parva, the second intermediate hosts of C. sinensis, which were naturally infected with $C$. sinensis, were caught in Shenyang city, Liaoning Province, People's Republic of China, which is an endemic area of clonorchiasis. Metacecariae of $C$. sinensis were collected by digesting fish with a pepsin- $\mathrm{HCl}(0.6 \%)$ artificial gastric juice. Five-week-old Sprague-Dawley rats were individually infected orally with 50 metacercariae. Eight weeks post infection, C. sinensis adult worms were recovered from bile ducts and homogenized in phosphate-buffered saline on ice, centrifuged for $30 \mathrm{~m}$ at 13000 r.p.m. and $4{ }^{\circ} \mathrm{C}$, and filtered using a syringe-driven $0.2-\mu \mathrm{m}$ syringe filter. Protein levels in the CA were measured using a Pierce BCA protein assay kit (Thermo, Rockford, IL, USA).

\section{Cell culture and antigen stimulation}

Murine DCs were cultured in Roswell Park Memorial Institute-1640 media supplemented with $10 \%$ fetal bovine serum (Gibco, Rockville, MD, USA), penicillin $\left(100 \mu \mathrm{g} \mathrm{ml}^{-1}\right.$; Gibco), streptomycin (100 $\mathrm{U} \mathrm{ml}^{-1}$, Gibco), $50 \mu \mathrm{M} \beta$-mercaptoethanol (Sigma-Aldrich, St Louis, MO, USA) and L-glutamine. DC2.4 cells were grown at $37^{\circ} \mathrm{C}$ in a humid environment with $5 \% \mathrm{CO}_{2}$. Bone marrows were aseptically collected from femurs and tibias of 4-12-week-old naive C57BL/6 mice, which were purchased from Sam Tako (Gyeonggi-do, Republic of Korea). Mice were humanely killed by cervical dislocation, and bone marrow cells were recovered and flushed from femurs using complete medium (Dulbecco's modified Eagle's medium, Gibco) supplemented with $5 \%$ fetal bovine serum (Gibco), 2 mm L-glutamine, $100 \mathrm{~g} \mathrm{ml}^{-1}$ penicillin (Gibco) and $100 \mathrm{U} \mathrm{ml}^{-1}$ streptomycin (Gibco) on ice. Single cells were counted and resuspended at $2 \times 10^{5}$ cells per $\mathrm{ml}$ in $10 \mathrm{ml}$ Dulbecco's modified Eagle's media supplemented with $10 \%$ fetal bovine serum, $100 \mu \mathrm{g} \mathrm{ml}^{-1}$ penicillin, $100 \mathrm{U} \mathrm{ml}^{-1}$ streptomycin, $2 \mathrm{~mm}$ L-glutamine, $50 \mu \mathrm{m} 2$-mercaptoethanol and $20 \mathrm{ng} \mathrm{ml}^{-1}$ of recombinant mouse granulocyte-macrophage colony-stimulating factor (Biosoure, Invitrogen, Carlsbad, CA, USA) and plated in bacteriological petri dishes. An additional $10 \mathrm{ml}$ of $20 \mathrm{ng} \mathrm{ml}^{-1}$ GM-CSF-containing medium was added on day 3 , and medium was replaced on day 6. On day 7, non-adherent DCs were collected, counted and seeded into six-well cell culture plates at $1 \times 10^{6}$ cells per $\mathrm{ml}$ for further analysis. Immature bone marrow-derived DCs (BMDCs) and DC2.4 cells were cultured with or without C. sinensis CA for the indicated duration. Cells and supernatants were collected and assayed for cell surface markers and cytokine production using enzyme-linked immunosorbent assay. ERK activation inhibitor peptide I ( 2 and $5 \mu \mathrm{M}$; Calbiochem, Laeufelfingen, Switzerland) were used to inhibit the ERK pathway. Animal experiments were conducted according to the guidelines of Seoul National University Institutional Animal Care and Use Committee (SNU-100117-1)

\section{Flow cytometric analysis}

After stimulating with Ag, adhesive DCs were collected by treatment with trypsin, washed with phosphate-buffered saline containing $0.05 \% \mathrm{NaN}_{3}$ and stained with FITC Hamster anti-mouse CD11c (BD-557400), FITC-conjugated Hamster anti-mouse CD80 (B7-1), monoclonal antibody (BD-553768), PE rat anti-mouse CD86 (BD-553692), PE rat anti-mouse I-A/I-E (BD-557000), FITC rat anti-mouse CD40 (BD-553790) (BD Biosciences, Franklin Lakes, NJ, USA), and FITC-TLR2 (sc-52736) and PE-TLR4 (sc-13591) (Santa Cruz Biotechnology, Santa Cruz, CA, USA) for $30 \mathrm{~m}$ in the dark, washed with phosphate-buffered saline and analyzed using FACS Calibur multicolor flow cytometry. Data were analyzed using CellQuest software (Becton-Dickinson, Franklin Lakes, NJ, USA).

\section{Cytokine enzyme-linked immunosorbent assay}

Sandwich enzyme-linked immunosorbent assays were used to measure the levels of IL-4, IL-5, IL-6, IL-10, IL-12, IL-13, interferon- $\gamma$, 
TNF- $\alpha$ and TGF- $\beta$ in culture supernatants. To assess the level of these cytokines in culture supernatants, we used commercial ELISA kits (eBioscience, San Diego, CA, USA) according to the manufacturer's instructions.

\section{Protein extraction and western blot analysis}

After stimulation with $C$. sinensis CA, DCs were collected and total protein was extracted from cell lysates using radioimmunoprecipitation assay buffer ( $1 \%$ Nonidet P-40; $0.2 \% \mathrm{Na}$ deoxycholate in a buffer containing $120 \mathrm{~mm} \mathrm{NaCl}, 0.2 \%$ SDS and $20 \mathrm{~mm}$ Tris, $\mathrm{pH} 8.0$ ) containing $1 \mathrm{~mm}$ phenylmethylsulfonyl fluoride and protease and phosphatase inhibitor cocktails (Sigma-Aldrich), incubated on ice for $30 \mathrm{~min}$, and centrifuged at 13000 r.p.m. for $10 \mathrm{~min}$ at $4{ }^{\circ} \mathrm{C}$. Supernatants were transferred to clean tubes, subjected to electrophoresis using a 10\% SDS-polyacrylamide electrophoresis gel and electroblotted onto polyvinylidene fluoride membranes. After blocking the membranes, proteins were probed with polyclonal or monoclonal antibodies specific to phospho-ERK1/2 (sc-7383), phospho-p38 (sc-7973), and phospho-JNK (sc-6254), total-ERK1/2 (sc-94), p38 (sc-535), JNK (sc-571) and actin (sc-1616) (Santa Cruz Biotechnology). Anti-goat, anti-rabbit and anti-mouse IgG antisera conjugated with horseradish peroxidase (Dako, Glostrup, Denmark) were used at a dilution of 1:2000 with 5\% skim milk and visualized using ECL Western blotting detection reagents (Amersham, Piscataway, NJ, USA).

\section{Statistical analysis}

Student's $t$-tests were used to determine the statistical significance of the results obtained. $P<0.05$ was considered to be significant.

\section{RESULTS}

Morphological changes of DC2.4 cells

Morphology of the mouse DC line DC2.4 was observed using microscopy after a 24-h incubation with $20 \mu \mathrm{g} \mathrm{ml}^{-1}$ of CA from C. sinensis. The group treated with CA had a 'spiked' morphology, which is characteristic of activated DCs, compared with the nontreated group (Figure 1).

\section{C. sinensis CA regulates cytokine production in DCs}

In light of these results, our attention focused on the analysis of cytokines that may modulate the activation of DCs. Th1-type cytokines (IL-12 and interferon- $\gamma$ ) were secreted at low concentrations (Figure 2), and the level of secreted Th2 cytokines (IL-4, IL-5 and IL-13) was barely detectable in both groups (data not shown). In addition, we did not find significant changes in the levels of IL- 6 and TNF- $\alpha$ secretion following the addition of CA (Figure 2). However, secretion of anti-inflammatory cytokines IL-10 and TGF- $\beta$ increased in DC2.4 cells (Figure 2). We compared cytokine levels with CA and LPS as a positive control and found that, in case of LPS treatment, IL-6 and TNF- $\alpha$ were significantly increased and no anti-inflammatory cytokine was produced (Figure 2).

\section{Generation of BMDCs and production of cytokines}

Next, we asked whether these findings are restricted to the DC2.4 cell line. To this end, cells isolated from the bone
A
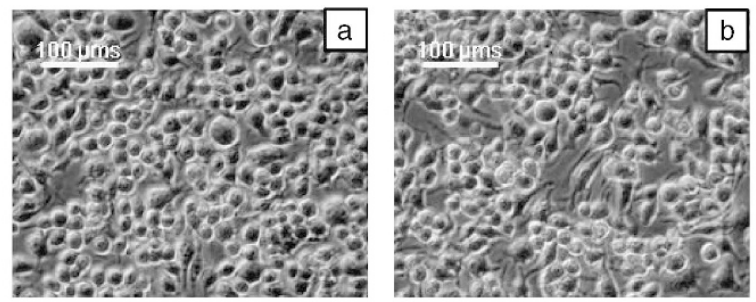

B
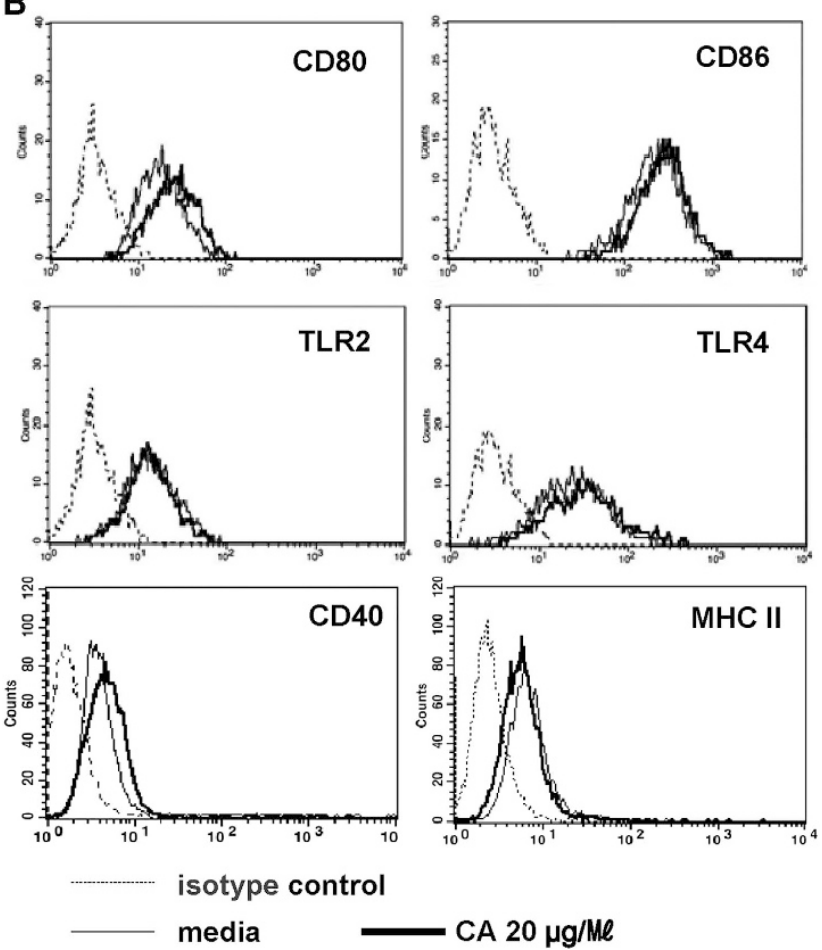

Figure 1 Panel A shows Morphological changes of DC2.4 cells treated with (a) medium, (b) $20 \mu \mathrm{g} \mathrm{ml}^{-1}$ of crude antigen (CA). Cells were plated in six-well plates $\left(2 \times 10^{5}\right.$ cells per well). After incubation for $24 \mathrm{~h}$, changes in cellular morphology in each group were determined using microscopy. Images were acquired using a digital camera at $\times 200$ magnification. This figure shows representative features of four independent experiments. Panel B shows no differences in levels of cell surface markers (CD80, CD86, Toll-like receptor (TLR)2 and TLR4) after treatment with $C$. sinensis crude antigen.

marrow were cultured for 7 days in medium containing $20 \mathrm{ng} \mathrm{ml}^{-1}$ granulocyte-macrophage colony-stimulating factor. Almost $80 \%$ of the cells were converted into CD11c-positive DCs (data not shown).

As demonstrated using the DC2.4 cell line system, BMDCs were also able to induce production of the anti-inflammatory cytokines IL-10 and TGF- $\beta$ (Figure 3 ). In addition, BMDCs did not upregulate Th1 cytokines (IL-12 and interferon- $\gamma$ ) and proinflammatory cytokines (IL-6, TNF- $\alpha$; Figure 3), and production of Th2 cytokines (IL-4, IL-5 and IL-13) was not detected after addition of CA from $C$. sinensis (data not shown). 


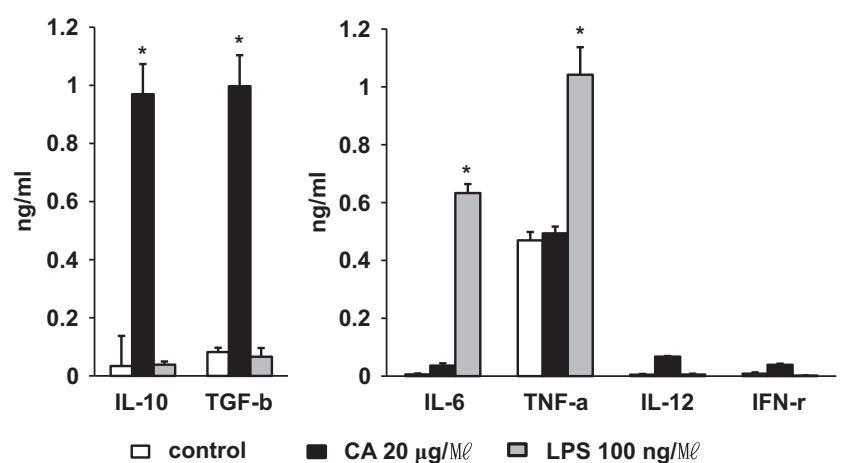

Figure 2 Production of cytokines following treatment of DC2.4 cells with $C$. sinensis crude antigen (CA) and lipopolysaccharide (LPS). DC2.4 cells were incubated with phosphate-buffered saline, $20 \mu \mathrm{g} \mathrm{ml}^{-1}$ CA and $100 \mathrm{ng} \mathrm{ml}^{-1}$ LPS for $24 \mathrm{~h}$, supernatants were collected and expression levels of interleukin (IL)-10, transforming growth factor (TGF- $\beta$ ), IL-4, IL-5, IL-6, IL-13, IL-12, interferon (IFN)- $\gamma$ and TNF- $\alpha$ were analyzed by enzyme-linked immunosorbent assay (ELISA). Data represent the means of three independent experiments. ${ }^{*} P<0.01$ ( $t$-test) was considered statistically significant.
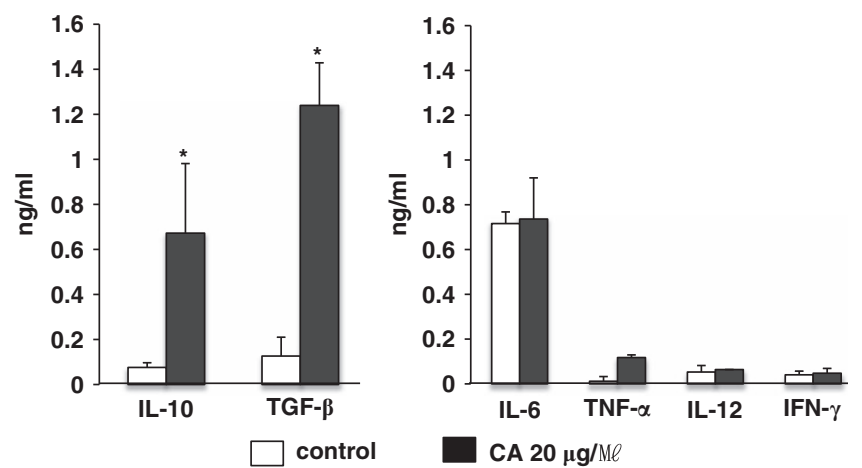

Figure 3 Production of cytokines following treatment of bone marrow-derived DCs (BMDC) cells with $C$. sinensis crude antigen (CA). BMDC cells were incubated with either phosphate-buffered saline or $20 \mu \mathrm{g} \mathrm{ml}^{-1}$ of $\mathrm{CA}$ for $24 \mathrm{~h}$, and the supernatant was collected to analyze the expression levels of interleukin (IL)-10, transforming growth factor (TGF)- $\beta$, IL-4, IL-5, IL-6, IL-13, IL- 12 , interferon (IFN) $-\gamma$ and TNF- $\alpha$ by enzyme-linked immunosorbent assay (ELISA). Data represent the means of three independent experiments. ${ }^{*} P<0.01$ ( $t$-test) was considered statistically significant.

\section{Expression of co-stimulatory molecules after treatment of} DC2.4 cells with $C$. sinensis CA

To evaluate changes in expression levels of activation-related molecules, DC2.4 cells were treated with $20 \mu \mathrm{g} \mathrm{ml}^{-1}$ C. sinensis $\mathrm{CA}$ for $24 \mathrm{~h}$, and flow cytometric analysis was used to analyze the expression levels of surface markers. As shown in Figure 1, levels of major histocompatibility complex II, CD40, CD80, CD86, TLR2 and TLR4 did not change following treatment with $C$. sinensis CA compared with the control group. These results were also observed at earlier $(12 \mathrm{~h})$ and later $(2$ days $)$ time points (data not shown).

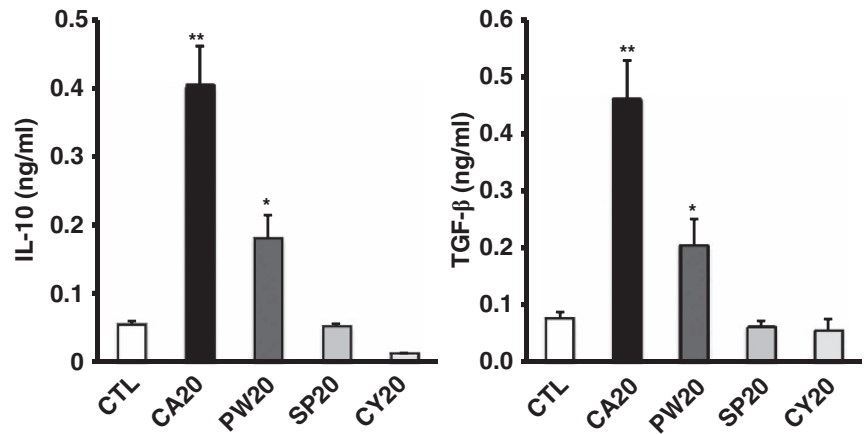

Figure 4 C. sinensis crude antigen (CA) regulates interleukin (IL)10 and transforming growth factor (TGF)- $\beta$ in dendritic cells (DCs) compared with other parasite antigens. DC2.4 cells were plated in six-well plates $\left(2 \times 10^{5}\right.$ cells per well). After treatment with different parasite antigens for $24 \mathrm{~h}$, including $C$. sinensis $C A$, $P$. westermani CA (PW), S. erinacei plerocercoid CA (SP) and $E$. granulosus hydatid cystic fluid (CY), supernatants were collected. Production of IL-10 and TGF- $\beta$ were determined using enzyme-linked immunosorbent assay (ELISA). Data represent the means of three independent experiments. ${ }^{*} P<0.05$ and ${ }^{* *} P<0.01$ ( $t$-test) were considered statistically significant.

\section{Regulation of IL-10 and TGF- $\beta$ expression in response to} parasite antigens

As CA from C. sinensis upregulated expression of IL-10 and TGF- $\beta$, we investigated the effect of other parasite antigens on changes in cytokine gene expression. We prepared CAs from Paragonimus westermani, Spirometra erinacei plerocercoid-sparaganum and Echinococcus granulosus cystic fluid antigen, and we measured protein levels of the anti-inflammatory cytokines, IL-10 and TGF- $\beta$. As shown in Figure 4 , we observed increases in IL- 10 and TGF- $\beta$ production in mouse DC2.4 cells treated with trematode antigens C. sinensis and $P$. westermani. Production of anti-inflammatory cytokines was significantly higher in DC2.4 cells treated with $C$. sinensis antigen compared with $P$. westermani antigen. In addition, we found that the level of TGF- $\beta$ expression has not changed after treatment with cestode antigen S. erinacei plerocercoid-sparaganum and E. granulosus cystic fluid antigen.

\section{Activation of ERK in DC2.4 cells upon treatment with C. sinensis CA}

As $C$. sinensis $C A$ induced the release of anti-inflammatory cytokines IL-10 and TGF- $\beta$ in DC2.4 cells and BMDCs, we investigated the signaling cascade involved in this process. We first evaluated the levels of MAPK in the control and treatment groups. Whole-cell lysates were collected after treatment with $C$. sinensis $C A$ for 10,30 and $60 \mathrm{~min}$. Figure 5 shows upregulation of ERK1/2 phosphorylation in DC2.4 cells treated with C. sinensis CA compared with nontreated DC2.4 cells. However, activation of JNK and p38 was not observed under these same conditions (data not shown). 

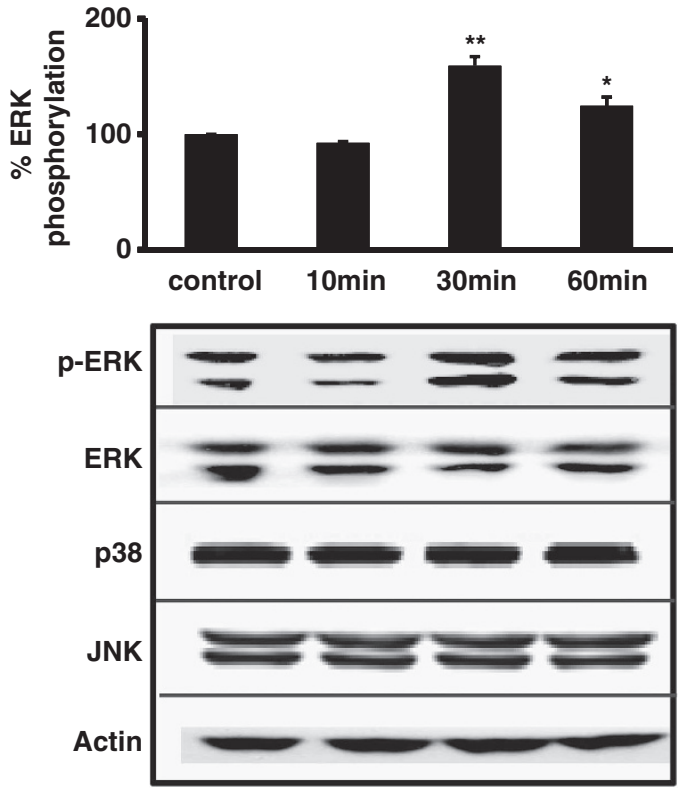

Figure 5 ERK1/2 phosphorylation in DC2.4 cells treated with C. sinensis crude antigen (CA). After treatment with $C$. sinensis $\mathrm{CA}$, whole-cell lysates were collected at 10, 30 and $60 \mathrm{~min}$. Phosphorylated ERK1/2, p38, JNK and total-ERK1/2, p38 and JNK levels were analyzed via western blot. Densitometry values were normalized to t-ERK, t-p38 or t-JNK, and then to the nontreated controls. Data represent the means of three independent experiments. ${ }^{*} P<0.05$ and ${ }^{* *} P<0.01$ ( $t$-test) were considered statistically significant.

\section{ERK is essential for upregulation of IL-10 and TGF- $\beta$ in} DC2.4 cells

We found that CA strongly induces IL-10 and TGF- $\beta$ secretion and activates ERK signals in DC2.4 cells. To verify that phosporylated ERK is responsible for the increased levels of anti-inflammatory cytokines, including IL-10 and TGF- $\beta$, we blocked this pathway using an ERK activation inhibitor peptide I. Figure 6 shows that ERK activation inhibitor peptide I is able to block CA-induced IL-10 and TGF- $\beta$ secretion.

\section{DISCUSSION}

As C. sinensis CA is in direct contact with host tissues and body fluids, it is likely that interactions between $C$. sinensis antigens and host cells elicit immune reactions. As DCs are key players in driving adaptive immunity, we examined the effects of CA on these cells. Results from the present study showing that DC cells produce regulatory cytokines demonstrate the immune suppressive effects of $C$. sinensis $\mathrm{CA}$ (Figures 2 and 3 ). This phenomenon was verified in the mouse DC line DC2.4 and in bone marrow-derived DCs.

Results from our investigation on the effects of CA on DC maturation support other studies that demonstrate that exposure of immature DCs to helminth-derived antigens induces a potent Treg immune response. ${ }^{13}$ However, results from Kane et al.,13 which showed that helminth-derived antigens can partially induce DC maturation, are not in
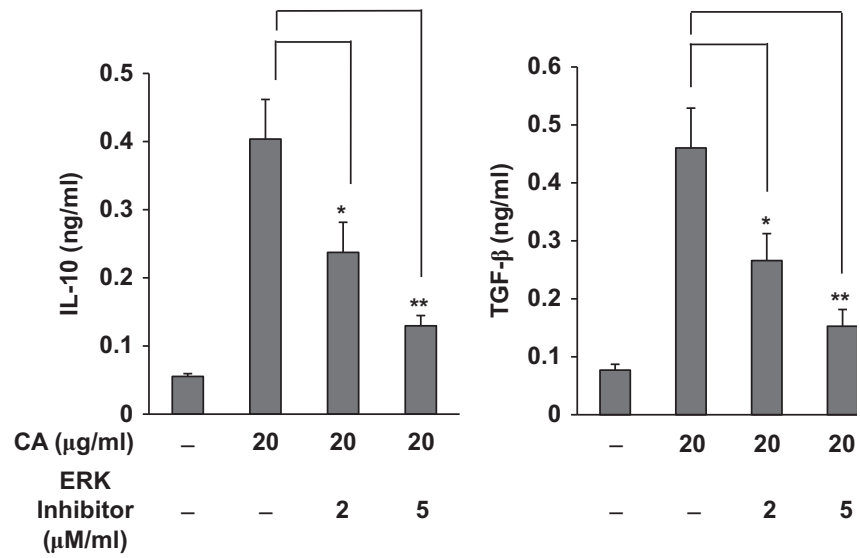

Figure 6 Effects of ERK activation inhibitor on crude antigen (CA)induced interleukin (IL)-10 and transforming growth factor (TGF)- $\beta$ levels. DC2.4 cells were cultured with 2 or $5 \mu \mathrm{m}$ of ERK activation inhibitor peptide I in phosphate-buffered saline and collected after incubation with $20 \mu \mathrm{g} \mathrm{ml}^{-1}$ of CA for $24 \mathrm{~h}$. Data represent the means \pm s.e. of three independent experiments. ${ }^{*} P<0.05$ and ${ }^{* *} P<0.01$ ( $t$-test) were considered statistically significant.

agreement with our results (Figure 1). Examples of helminth-derived antigens include the filarial-derived antigen ES- $62^{11,12}$ and the S. mansoni larvae antigen. ${ }^{13}$

In this study, we found that DC secreted TGF- $\beta$ and IL- 10 . TGF- $\beta$ is known to act as a multifunctional cytokine involved in cell growth and differentiation. ${ }^{18}$ For example, TGF- $\beta$ has been implicated in biliary epithelial proliferation in clinical and experimental biliary tract disease. ${ }^{19}$ In addition, IL-10 and TGF- $\beta$ were shown to be important in tolerance induction and immune regulation. Immature DCs have been shown to induce tolerance. ${ }^{20}$ Recently, a new regulatory subset of DCs, which have been reported in vivo, were shown to inhibit antigen-specific T-cell proliferation, have a Th2 cytokine profile, and exhibit increased secretion of IL-10 and decreased secretion of IL-12. ${ }^{21}$ Although we showed that CA upregulates IL-10 and TGF- $\beta$ in DC cells and BMDCs, we did not determine whether CA drives Th2 or T-regulatory immune responses. Preliminary in vivo observations suggest that $C$. sinensis infection induces Th2 responses in mouse model (data not shown). Nevertheless, our findings indicate that exposure to $\mathrm{CA}$, in addition to a subsequent lack of proinflammatory cytokine production and absence of costimulatory molecules that are important for DC activation, may interfere with activation of DCs and their ability to prime naive $\mathrm{T}$ cells.

Our data support previous findings demonstrating that C. sinensis infections suppress Th1 immune responses in vivo. ${ }^{17}$ Furthermore, suppression of Th1 responses is likely to be a survival mechanism, allowing the organism to stay over 25 years in the host without eliciting acute inflammation. Further investigation of the molecular mechanisms involved in C. sinensis-derived induction of regulatory DC in vitro may provide a better understanding of mechanisms used by parasites to evade or regulate the immune system. Future 
studies may also provide additional tools for developing new strategies for effective immunotherapy. In addition, it would be interesting to investigate whether Th1 responses are inhibited by IL-10 and TGF- $\beta$ produced from DCs upon $C$. sinensis infection in vivo. If the secretion of IL-10 and TGF- $\beta$ can suppress Th1 immune response, anti-inflammatory cytokines might be the key survival factor, allowing the liver fluke to stay over 25 years in the host without eliciting acute inflammation. It would be interesting to investigate whether IL-10 and TGF- $\beta$ secretion by DCs can regulate the immune system after infection with $C$. sinensis in vivo.

Considerable evidence links ERK activation to IL-10 induction in DCs. ${ }^{22,23}$ Moreover, it has been demonstrated that ERK activation can upregulate TGF- $\beta$ expression in DCs. ${ }^{24}$ Interestingly, our results indicate that ERK activation may be linked to upregulation of TGF- $\beta$ and IL-10 in mouse DCs. It would be of interest to determine whether TLR ligands, which stimulate multiple signals including ERK, induce TGF- $\beta$ upregulation in DCs.

In summary, this is the first study to report that $C$. sinensis CA modulates production of the anti-inflammatory cytokines IL-10 and TGF- $\beta$ in DCs. Furthermore, ERK signaling regulates these cytokines. Given the modular effects that CAs exhibit, understanding these mechanisms may lead to the development of novel immunotherapies for the treatment of Th1-mediated inflammatory diseases.

\section{ACKNOWLEDGEMENTS}

This work was supported by Korea Institute of Science and Technology Information (KISTI K-13-L01-C02-S04) and grants from Seoul National University Hospital Research Fund (grant number 800-2011-0393).

1 Kapsenberg ML. Dendritic-cell control of pathogen-driven T-cell polarization. Nat Rev Immunol 2003; 3: 984-993.

2 Banchereau J, Steinman RM. Dendritic cells and the control of immunity. Nature 1998; 392: 245-252.

3 Maizels RM, Yazdanbakhsh M. Immune regulation by helminth parasites: cellular and molecular mechanisms. Nat Rev Immunol 2003; 3: 733-744.

4 MacDonald AS, Maizels RM. Alarming dendritic cells for Th2 induction. J Exp Med 2008; 205: 13-17.

5 Qian C, Jiang X, An H, Yu Y, Guo Z, Liu S et al. TLR agonists promote ERKmediated preferential IL-10 production of regulatory dendritic cells (diffDCs), leading to NK-cell activation. Blood 2006; 108: 2307-2315.

6 Fallon PG, Richardson EJ, Smith P, Dunne DW. Elevated type 1, diminished type 2 cytokines and impaired antibody response are associated with hepatotoxicity and mortalities during Schistosoma mansoni infection of CD4-depleted mice. Eur J Immunol 2000; 30: 470-480.
7 Maizels RM, Balic A, Gomez-Escobar N, Nair M, Taylor MD, Allen JE. Helminth parasites-masters of regulation. Immunol Rev 2004; 201: 89-116.

8 Hamilton CM, Dowling DJ, Loscher CE, Morphew RM, Brophy PM, O'Neill SM. The Fasciola hepatica tegumental antigen suppresses dendritic cell maturation and function. Infect Immun 2009; 77: 2488-2498.

9 Wickelgren I. Immunotherapy. Can worms tame the immune system? Science 2004; 305: 170-171.

10 Harnett W, Harnett MM. Therapeutic immunomodulators from nematode parasites. Expert Rev Mol Med 2008; 10: e18.

11 Whelan M, Harnett MM, Houston KM, Patel V, Harnett W, Rigley KP. A filarial nematode-secreted product signals dendritic cells to acquire a phenotype that drives development of Th2 cells. J Immunol 2000; 164: 6453-6460.

12 Goodridge HS, Wilson EH, Harnett W, Campbell CC, Harnett MM, Liew FY. Modulation of macrophage cytokine production by ES-62, a secreted product of the filarial nematode Acanthocheilonema viteae. J Immunol 2001; 167: 940-945.

13 Kane CM, Cervi L, Sun J, McKee AS, Masek KS, Shapira S et al. Helminth antigens modulate TLR-initiated dendritic cell activation. J Immunol 2004; 173: 7454-7461

14 Flynn RJ, Mulcahy G. The roles of IL-10 and TGF-beta in controlling IL-4 and IFN-gamma production during experimental Fasciola hepatica infection. Int J Parasitol 2008; 38: 1673-1680.

15 Lun ZR, Gasser RB, Lai DH, Li AX, Zhu XQ, Yu XB et al. Clonorchiasis: a key foodborne zoonosis in China. Lancet Infect Dis 2005; 5: 31-41.

16 Kim TS, Cho SH, Huh S, Kong Y, Sohn WM, Hwang SS et al. A nationwide survey on the prevalence of intestinal parasitic infections in the Republic of Korea, 2004. Korean J Parasitol 2009; 47: 37-47.

17 Choi YK, Yoon BI, Won YS, Lee CH, Hyun BH, Kim HC et al. Cytokine responses in mice infected with Clonorchis sinensis. Parasitol Res 2003; 91: 87-93.

18 Derynck R, Akhurst RJ, Balmain A. TGF-beta signaling in tumor suppression and cancer progression. Nat Genet 2001; 29: 117-129.

19 Goggins M, Shekher M, Turnacioglu K, Yeo CJ, Hruban RH, Kern SE. Genetic alterations of the transforming growth factor beta receptor genes in pancreatic and biliary adenocarcinomas. Cancer Res 1998; 58: 5329-5332.

20 van Kooyk Y, Engering A, Lekkerkerker AN, Ludwig IS, Geijtenbeek TB. Pathogens use carbohydrates to escape immunity induced by dendritic cells. Curr Opin Immunol 2004; 16: 488-493.

21 Fujita S, Seino K, Sato K, Sato Y, Eizumi K, Yamashita N et al. Regulatory dendritic cells act as regulators of acute lethal systemic inflammatory response. Blood 2006; 107: 3656-3664.

22 Sato K, Nagayama H, Tadokoro K, Juji T, Takahashi TA. Extracellular signal-regulated kinase, stress-activated protein kinase/c-Jun $\mathrm{N}$-terminal kinase, and p38mapk are involved in IL-10-mediated selective repression of TNF-alpha-induced activation and maturation of human peripheral blood monocyte-derived dendritic cells. J Immunol 1999; 162: 3865-3872.

23 Agrawal A, Dillon S, Denning TL, Pulendran B. ERK1 -/ - mice exhibit Th1 cell polarization and increased susceptibility to experimental autoimmune encephalomyelitis. J Immunol 2006; 176: 5788-5796.

24 Escors D, Lopes L, Lin R, Hiscott J, Akira S, Davis RJ et al. Targeting dendritic cell signaling to regulate the response to immunization. Blood 2008; 111: 3050-3061.

This work is licensed under a Creative Commons attribution-NonCommercial-NoDerivs 3.0 Unported License. To view a copy of this license, visit http:// creativecommons.org/licenses/by-nc-nd/3.0/ 